\title{
Guanosine to Adenosine Transition Abnormality
}

National Cancer Institute

\section{Source}

National Cancer Institute. Guanosine to Adenosine Transition Abnormality. NCI

Thesaurus. Code C45641.

A point mutation involving the substitution of Adenosine (a purine base) for Guanosine (a purine base) in a DNA sequence from eukaryotic or prokaryotic organisms. This abnormality can be either heritable or occur somatically. 